\title{
Madm (Mlf1 adapter molecule) cooperates with Bunched A to promote growth in Drosophila
}

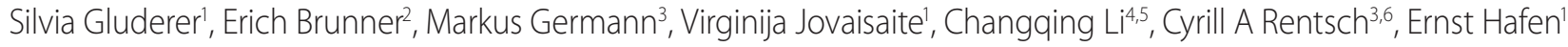 \\ and Hugo Stocker*1
}

See minireview at http://jbiol.com/content/9/1/8

\begin{abstract}
Background: The TSC-22 domain family (TSC22DF) consists of putative transcription factors harboring a DNAbinding TSC-box and an adjacent leucine zipper at their carboxyl termini. Both short and long TSC22DF isoforms are conserved from flies to humans. Whereas the short isoforms include the tumor suppressor TSC-22 (Transforming growth factor- $\beta 1$ stimulated clone-22), the long isoforms are largely uncharacterized. In Drosophila, the long isoform Bunched A (BunA) acts as a growth promoter, but how BunA controls growth has remained obscure.

Results: In order to test for functional conservation among TSC22DF members, we expressed the human TSC22DF proteins in the fly and found that all long isoforms can replace BunA function. Furthermore, we combined a proteomics-based approach with a genetic screen to identify proteins that interact with BunA. Madm (Mlf1 adapter molecule) physically associates with BunA via a conserved motif that is only contained in long TSC22DF proteins. Moreover, Drosophila Madm acts as a growth-promoting gene that displays growth phenotypes strikingly similar to bunA phenotypes. When overexpressed, Madm and BunA synergize to increase organ growth.
\end{abstract}

Conclusions: The growth-promoting potential of long TSC22DF proteins is evolutionarily conserved. Furthermore, we provide biochemical and genetic evidence for a growth-regulating complex involving the long TSC22DF protein BunA and the adapter molecule Madm.

\section{Background}

A prevalent model of carcinogenesis suggests that sequential activation of oncogenes and inactivation of tumor suppressor genes occur in a multistep process leading to deviant growth. Over the past decades much effort has been put into identifying tumor suppressor genes and their pathways because they represent attractive drug targets for cancer therapy. On the basis of expression data derived from various human and murine tumor tissues, Transforming growth factor- $\beta 1$ stimulated clone-22 (TSC-22) - originally identified as a TGF- $\beta 1$ responsive gene [1] - is believed to be a tumor suppressor gene [2-5]. TSC-22 exhibits pro-apoptotic functions in cancer cell lines [6,7], and a recent study reported that genetic disruption of the TSC-22 gene in mice causes

*Correspondence: stocker@imsb.biol.ethz.ch

'Institute of Molecular Systems Biology, ETH Zurich, Wolfgang-Pauli-Strasse 16, 8093 Zurich, Switzerland

Full list of author information is available at the end of the article higher proliferation and repopulation efficiency of hematopoietic precursor cells, consistent with a role of TSC-22 in tumor suppression [8]. However, TSC-22 knockout mice do not display enhanced tumorigenesis. Because TSC-22 possesses a leucine zipper and a novel motif capable of binding DNA in vitro - the TSC-box [9] TSC-22 is likely to operate as a transcription factor. Alternatively, TSC-22 might act as transcriptional regulator as it binds to Smad4 via the TSC-box and modulates the transcriptional activity of Smad4 [10]. Furthermore, Fortilin (TCTP) binds to and destabilizes TSC-22, thereby impeding TSC-22-mediated apoptosis [11].

Unraveling the precise mechanism by which TSC-22 acts is demanding because there are several mammalian genes homologous to TSC-22 that could have, at least in part, redundant functions. TSC-22 is affiliated with the TSC-22 domain family (TSC22DF) consisting of putative transcription factors that are characterized by a carboxyterminal leucine zipper and an adjacent TSC-box. This protein family is conserved from Caenorhabditis elegans 
to humans and is encoded by four separate loci in mammals, TSC22D1 to TSC22D4. These loci produce several isoforms that can be subdivided into a short and a long class depending on the length of the isoformspecific amino-terminal sequences and depending on the presence of two conserved, as-yet-uncharacterized motifs in the amino-terminal part of the long isoforms $[12,13]$. In addition to the (partial) redundancy, synergistic and/ or antagonistic functions among TSC-22 (TSC22D1.2) and its homologs are likely as TSC22DF proteins can form heterodimers [13] and may compete for common binding partners or target genes.

The short class of TSC22DF variants, including TSC-22 (TSC22D1.2), is well studied. In mice, TSC22D2 produces several short transcripts that are important for the osmotic stress response of cultured murine kidney cells [14]. TSC22D3v2, also known as Gilz (glucocorticoid-induced leucine zipper), is required in the immune system for T-cell receptor mediated cell death [15-18]. Moreover, Gilz is a direct target gene of the transcription factor FoxO3 [19], and several binding partners of the Gilz protein are known, including NF- $\mathrm{kB}$, c-Jun, c-Fos and Raf-1 [20-22]. In addition, short isoforms encoded by TSC22D 3 have differential functions in the aldosterone response, sodium homeostasis and proliferation of kidney cells [23].

The function of long TSC22DF members is less well understood. The long isoform TSC22D1.1, produced by the TSC-22 locus, as well as the long human TSC22D2 protein are largely uncharacterized. TSC22D4 is important for pituitary development [24] and can form heterodimers with TSC-22 (TSC22D1.2) [13]. Functional in vivo studies on TSC22DF, especially on the long isoforms, are needed to clarify how TSC-22 (TSC22D1.2) can act as a tumor suppressor.

Drosophila melanogaster is a valuable model organism for investigating the function of TSC22DF proteins in growth regulation for two reasons. First, many tumor suppressor genes [25] and growth-regulating pathways $[26,27]$ have been successfully studied in the fly. Second, the Drosophila genome contains a single locus, bunched (bun), encoding three nearly identical long and five short isoforms of TSC22DF members (FlyBase annotation FB2009_05 [28]). Thus, the redundancy and complexity of interactions among TSC22DF proteins are markedly lower in Drosophila than in mammals. Drosophila bun is important for oogenesis, eye development and the proper formation of the embryonic peripheral nervous system [29-31]. Furthermore, bun is required for the development of $\alpha / \beta$ neurons of the mushroom body, a brain structure involved in learning and memory [32]. It has been proposed that bun acts as a mitotic factor during the development of $\alpha / \beta$ neurons.

Two studies that we and others carried out $[12,33]$ have demonstrated that, in addition to its role in patterning processes, bun plays a crucial role in growth regulation. Whereas the long Bun isoforms are positive growth regulators, genetic disruption of the short transcripts bunB-E and bunH does not alter growth. However, overexpression of bunB and bunC does interfere in a dominant-negative manner with normal bunA function. These results on Drosophila bun apparently contradict data describing mammalian TSC-22 as a growthsuppressing gene. To resolve this conflict, we hypothesized that the as-yet-uncharacterized long TSC-22 isoform (TSC22D1.1) is a functional homolog of BunA in growth regulation and that it is antagonized by the short isoform TSC22D1.2.

Here we investigate the evolutionary functional conservation between BunA and the human TSC22DF proteins. We report that long TSC-22 (TSC22D1.1) as well as the long human isoforms TSC22D2 and TSC22D4 can substitute for BunA function but the short isoforms cannot. In addition, we demonstrate that the growthpromoting function of BunA is - at least in part mediated by Mlf1 adapter molecule (Madm). We have identified Madm in a genetic screen for growth regulators as well as in a proteomic screen for BunA-interacting proteins, and we show that BunA and Madm cooperate in promoting growth during development.

\section{Results \\ Long human TSC22DF proteins can substitute for BunA in Drosophila}

We hypothesized that the long isoform encoded by the TSC-22 locus, TSC22D1.1, is a functional homolog of BunA with growth-promoting capacity, and that it is antagonized by the short isoform TSC22D1.2. Therefore, we tested whether human TSC22D1.1 or any other TSC22DF member is able to replace BunA function in Drosophila. The UAS/Gal4 expression system [34] was combined with a site-specific integration system [35] to express the TSC22DF members. Ubiquitous expression of the long - but not of the short - human TSC22DF isoforms (Figure 1a) resulted in a rescue of the lethality of bun mutants carrying a deletion allele $(200 B)$ that is likely to be null for all bun isoforms [12] (Figure 1b). Thus, TSC22D1.1 has the ability to replace BunA function in the fly whereas TSC22D1.2 does not. Furthermore, all long human TSC22DF isoforms can act in place of BunA in Drosophila, suggesting that sequences conserved in the long isoforms enable BunA to promote growth.

\section{Madm (MIf1 adapter molecule) interacts biochemically with BunA}

How BunA exerts its growth-regulating function is unknown. It is conceivable that a protein specifically binding to long TSC22DF isoforms accounts for the growth-promoting ability. Therefore, we set out to identify 


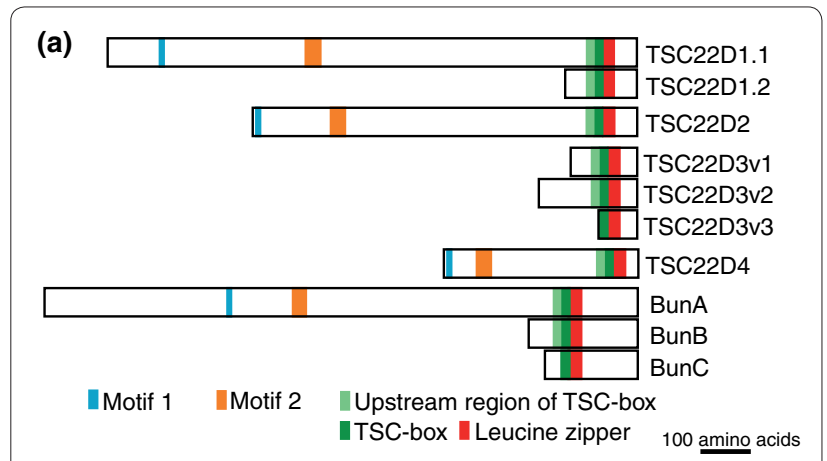

(b)

\begin{tabular}{|c|c|c|c|c|}
\hline & $\begin{array}{c}\text { Leaky } \\
\text { expression }\end{array}$ & Arm-Gal4 & Da-Gal4 & Act5C-Gal4 \\
\hline UAS-TSC22D1.1 & $0 \%$ & $39 \%$ & $85 \%$ & $1 \%$ \\
\hline UAS-TSC22D1.2 & $0 \%$ & $0 \%$ & $0 \%$ & $0 \%$ \\
\hline UAS-TSC22D2 & $0 \%$ & $50 \%$ & $26 \%$ & $3 \%$ \\
\hline $\begin{array}{l}\text { UAS-TSC22D3 } \\
\text { v1-3 }\end{array}$ & $0 \%$ & $0 \%$ & $0 \%$ & $0 \%$ \\
\hline UAS-TSC22D4 & $0 \%$ & $46 \%$ & $125 \%$ & $3 \%$ \\
\hline UAS-bunA ORF & $\begin{array}{l}11 \%(1 \mathrm{c}) \\
81 \%(2 \mathrm{c})\end{array}$ & $11 \%$ & $0 \%$ & $0 \%$ \\
\hline UAS-bunA cDNA & $\begin{array}{l}12 \%(1 \mathrm{c}) \\
26 \%(2 \mathrm{c})\end{array}$ & $117 \%$ & $17 \%$ & $0 \%$ \\
\hline $\begin{array}{l}\text { UAS-bunA cDNA } \\
\text { insertion on III }\end{array}$ & $0 \%$ & $82 \%$ & $145 \%$ & $2 \%$ \\
\hline $\begin{array}{l}\text { UAS-bunB cDNA } \\
\text { insertion on III }\end{array}$ & $0 \%$ & $0 \%$ & $0 \%$ & $0 \%$ \\
\hline $\begin{array}{l}\text { UAS-bunC cDNA } \\
\text { insertion on III }\end{array}$ & $0 \%$ & $0 \%$ & $0 \%$ & $0 \%$ \\
\hline
\end{tabular}

Figure 1. Long human TSC22DF isoforms can replace BunA function in Drosophila. (a) Schematic drawing of human and Drosophila TSC22DF proteins that were tested for their ability to rescue the lethality of bun mutants. The long isoforms possess two short conserved stretches named motif 1 and motif 2 . Whereas BunA represents the long TSC22DF isoforms in Drosophila, BunB and BunC are two of the short isoforms. (b) Expression of long TSC22DF isoforms restores the viability of bun mutants. The quality of the rescue is indicated as a percentage of the expected Mendelian ratio. The Gal 4 driver lines are ordered according to the strength of ubiquitous expression they direct during development, with armGal4 being the weakest and Act5C-Gal4 the strongest driver line. In each experimental cross, $n \geq 200$ progeny flies were analyzed. Leaky expression, without Gal4; 1 c and 2 c, one or two copies of the respective UAS construct. The $\mathrm{ZH}$-attP-86Fb integration site seems to mediate strong expression as the UAS-attB-bunA constructs (ORF and CDNA) do not need to be driven by a Gal4 line for rescue, in contrast to the UAS-bunA construct (CDNA) generated by standard P-elementmediated germline transformation (inserted non-site-specifically on chromosome III). Note that too high expression of long TSC22DF members is harmful to flies. In a wild-type background, Act5CGal4-directed expression ( $n \geq 200$ ) of TSC22D2 and of bunA ORF kills the animals ( $0 \%$ survival). Expression from the bunA cDNA construct produces few escapers (3\%), whereas expression from the bunA cDNA P-element construct and of TSC22D4 results in semi-viability (14\% and 69\%, respectively). Only TSC22D 1.1 can be expressed by Act5C-Gal4 without compromising survival (>80\%). Thus, there appears to be an optimal range of long TSC22DF concentration for viability.

binding partners by means of pulldown experiments combining affinity purification and mass spectrometry
(AP-MS) [36,37]. As baits, we expressed green fluorescent protein (GFP)- or hemagglutinin (HA)-tagged versions of the full-length BunA protein (rather than BunA-specific peptides, which might not preserve the three-dimensional structure of BunA) in Drosophila S2 cells and affinity purified the protein complexes by means of anti-GFP or anti-HA beads, respectively. The purified complexes were analyzed by tandem mass spectrometry (LC-MS/MS), and the proteins identified were judged as good candidates if they satisfied the following three criteria: they were not found in control experiments (HA-tagged GFP was used as bait and affinity purified using anti-GFP or anti-HA beads); they showed up in several independent AP-MS experiments; and they had an identification probability above an arbitrary threshold (Mascot score 50). We identified the adapter protein Madm as a good candidate in two independent experiments [see Additional file 1].

To confirm the binding between Madm and BunA, inverse pulldown assays using HA-Madm as bait were carried out in S2 cells. Endogenous BunA co-immunoprecipitated with HA-tagged Madm expressed under the control of a metallothionein-inducible promoter (Figure 2a). Moreover, BunA showed up as putative Madm binding partner in an AP-MS experiment [see Additional file 1].

Assuming that BunA and Madm interact, they should at least partially co-localize. Immunofluorescence studies in S2 cells revealed that GFP-BunA and HA-Madm signals in fact largely overlapped (Figure 2b,c). Interestingly, the HA-Madm signal was less dispersed when GFP-BunA was expressed in the same cell, indicating that the interaction with BunA altered the subcellular localization of HA-Madm (Figure 2c). A statistical analysis (Materials and methods) revealed that HA-Madm was only localized in punctae when co-overexpressed with GFP-BunA $(100 \%, n=50)$ but not when co-overexpressed with GFP $(0 \%, n=50)$. Moreover, when a mutated HA-Madm protein ( $\mathrm{R} 525 \mathrm{H}$, see below) was expressed, the localization in punctae was lost in $66 \%$ of cells co-overexpressing GFP-BunA $(n=50)$. The GFP-BunA signal largely overlapped with the Golgi marker GMAP210 [38] but not with an endoplasmic reticulum (ER) marker (Figure 2d, and data not shown), indicating that GFP-BunA localizes to the Golgi. The localization of BunA and Madm was not dependent on their tag because GFP- and HA-tagged BunA and Madm behaved similarly (data not shown). Furthermore, GFP-tagged BunA and Madm proteins were functional because they rescued the lethality of bun and Madm mutants, respectively, when expressed in the fly (Materials and methods). Taken together, our AP-MS and co-localization studies demonstrate that the adapter molecule Madm associates with BunA. 


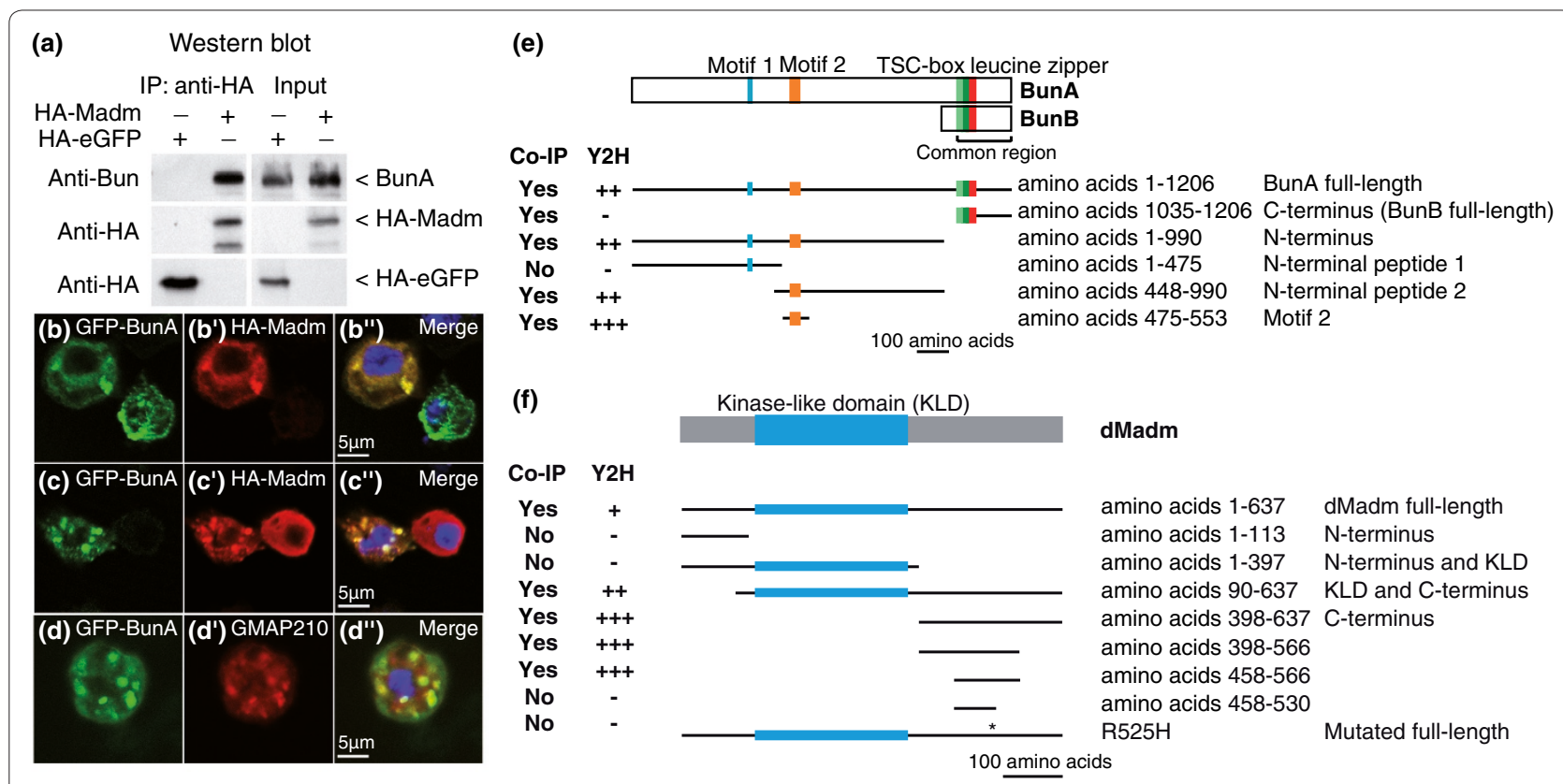

Figure 2. Madm interacts biochemically with BunA. (a) Western blot showing that endogenous BunA is pulled down together with HA-Madm. Anti-HA beads were used to capture either HA-Madm or HA-eGFP as a negative control, respectively. A tenth of the cell lysate was used for the input control. (b,c) Co-localization studies of BunA and Madm in Drosophila S2 cells. In (b-b") a stable cell line capable of producing GFP-BunA in every cell was transiently transfected with a plasmid leading to expression of HA-Madm in a subset of cells (and vice versa in c-c"). Co-overexpression of GFP-BunA influences the localization of HA-Madm, resulting in a less dispersed pattern (c-c"). (d) GFP-BunA co-localizes with the Golgi marker GMAP210 (Golgi microtubule-associated protein of 210 kDa) [38]. (e,f) Schematic drawing of BunA (e) and Madm (f) constructs tested in $\mathrm{Y} 2 \mathrm{H}$ and co-IP assays for an interaction with full-length Madm and BunA, respectively. The results of the Y2H and co-IP experiments are summarized on the left [see Additional files 2 and 3 for the primary results]. The physical interaction of BunA and Madm is mediated by a short protein sequence encompassing the conserved motif 2 in BunA and a carboxy-terminal sequence in Madm, respectively [see Additional file 4 for alignments].

\section{Madm binds to a long-isoform-specific sequence in BunA}

To investigate whether Madm binds to long-isoformspecific sequences, we mapped the Madm-binding region in BunA, and vice versa, by means of co-immunoprecipitation (co-IP) and yeast two-hybrid (Y2H) experiments. The advantage of the $\mathrm{Y} 2 \mathrm{H}$ system is that Drosophila bait proteins are unlikely to form complexes or dimers - in case of BunA via its leucine zipper - with endogenous yeast proteins and therefore the observed $\mathrm{Y} 2 \mathrm{H}$ interactions are presumably direct. Our co-IP and $\mathrm{Y} 2 \mathrm{H}$ data indicated that a long-isoform-specific aminoterminal sequence of BunA (amino acids 475-553) encompassing motif 2 is sufficient for the interaction with Madm (Figure 2e and Additional file 2). Moreover, one of the two point mutations isolated in a genetic screen that affect motif 2 (the hypomorphic bun alleles $A-R 508 \mathrm{~W}$ and A-P519L; see Additional data file 4 and [12]) weakened the binding to Madm.

The BunA-binding domain in Drosophila Madm was reciprocally mapped by means of co-IP and $\mathrm{Y} 2 \mathrm{H}$ experiments to the carboxy-terminal amino acids 458566 (Figure 2f and Additional file 3). Furthermore, we found that amino acids 530-566, including a nuclear export signal (NES) and a predicted nuclear-receptorbinding motif (LXXLL) in mammals, were not dispensable for the binding to BunA [see Additional file 4]. In addition, a point mutation leading to the arginine to histidine substitution $\mathrm{R} 525 \mathrm{H}$ disrupted BunA-binding (the point mutation derived from the Madm allele 4S3; Figure 3e). Thus, Madm is a Bun-interacting protein that specifically binds the long Bun isoforms.

\section{Drosophila Madm is a growth-promoting gene}

In a parallel genetic screen based on the eyFLP/FRT recombinase system, we were searching for mutations that cause growth phenotypes akin to the bunA phenotype [12]. A complementation group consisting of seven recessive lethal mutations was mapped to the Madm genomic locus (Materials and methods). The seven ethyl methanesulfonate (EMS)-induced mutations caused a small head (pinhead) phenotype; therefore, the affected gene encodes a positive growth regulator (Figure $3 \mathrm{~b}, \mathrm{c}$ ). The rather compact genomic locus of Madm contains two exons and produces a single protein isoform (Figure 3e). The adapter protein Madm possesses a kinase-like domain that lacks the conserved ATP-binding motif, thus 


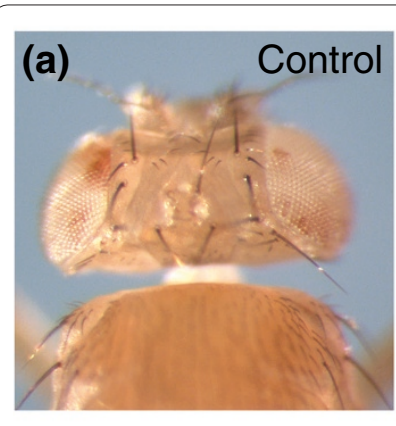

(e)

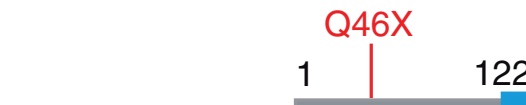

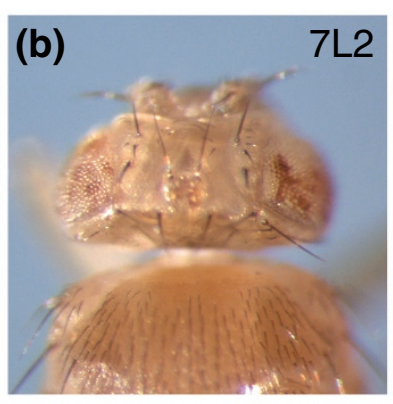
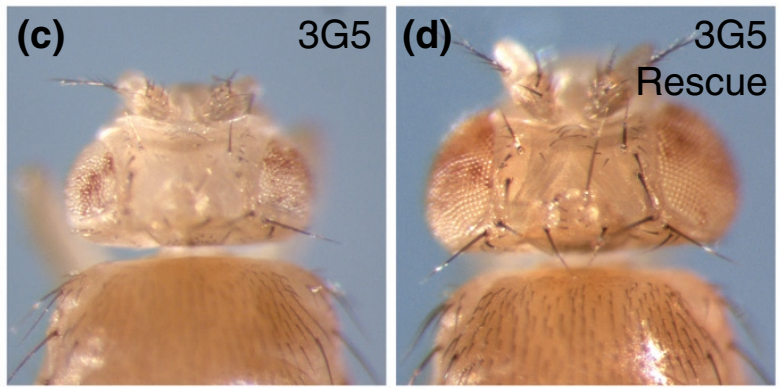

$4 \mathrm{~S} 3$

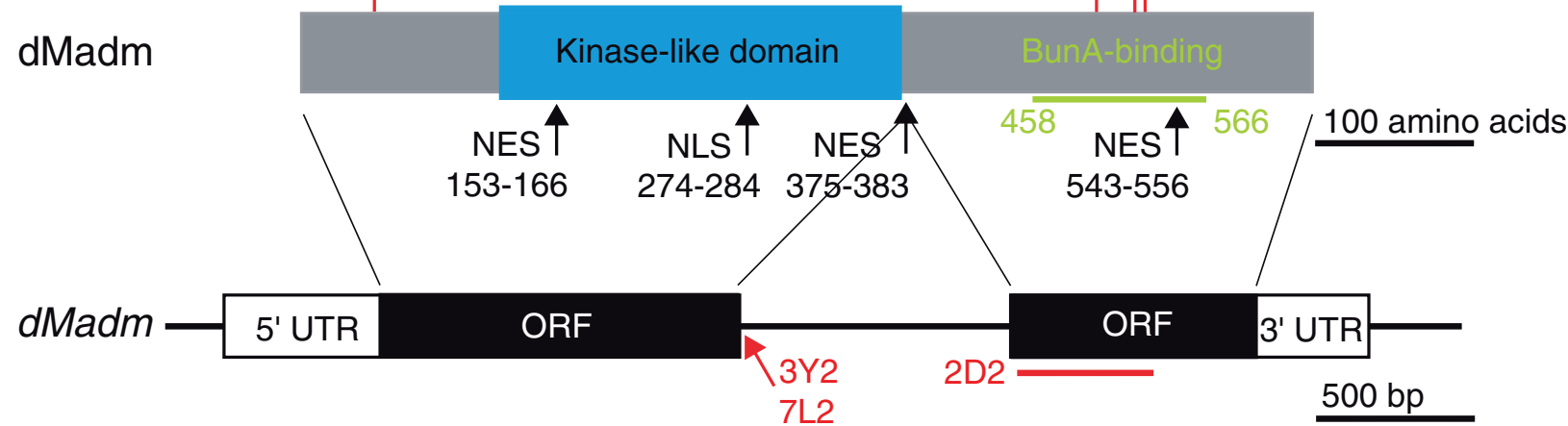

Figure 3. A genetic eyFLP/FRT-based screen in Drosophila identifies Madm as a positive growth regulator. (a-d) Dorsal view of mosaic heads generated by means of the eyFLP/FRT system. (a) The isogenized FRT82 chromosome used in the genetic screen produces a control mosaic head. $(b, c)$ Heads largely homozygous mutant for an EMS-induced Madm mutation display a pinhead phenotype that can be reverted by one copy of a genomic Madm rescue construct (d). (e) Graphic representation of the Drosophila Madm protein (top) and gene (bottom). In the protein, the BunA-binding region and the NES and NLS sequences are indicated (netNES 1.1 [63], ELM [64], PredictNLS [65]). The seven alleles isolated in the genetic screen and the sites of their EMS-induced mutations are in red. Amino acid changes in the protein are indicated. In alleles $3 Y 2$ and $7 L 2$, the first nucleotide downstream of the first Madm exon is mutated, thus disrupting the splice donor site. In allele 2D2, a deletion causes a frameshift after amino acid 385, resulting in a premature translational stop after an additional 34 amino acids. Alleles 3Y2, 4S3, and 7L2 lead to a pinhead phenotype of intermediate strength (b) whereas $2 \mathrm{D} 2,2 \mathrm{U} 3$, and $3 \mathrm{G} 5$ produce a stronger pinhead phenotype (c). The hypomorphic allele $3 T 4$ generates a weak pinhead phenotype (data not shown). Genotypes of the flies shown are: (a) $y, w$, eyFlp/y, $w ; F R T 82 B / F R T 82 B, w^{+}, C^{\beta B 3} ;(b, c) y, w$,

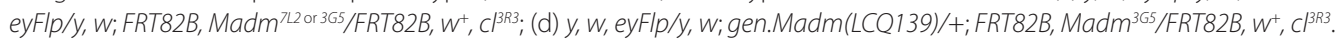

rendering it a non-functional kinase $[39,40]$. Moreover, Drosophila Madm carries several conserved NESs and a non-conserved nuclear localization signal (NLS; Figure 3e) [40]. We identified molecular lesions in all seven EMSinduced mutations (six point mutations and one deletion; Figure 3e) by sequencing the Madm open reading frame (ORF). Expression of a genomic Madm as well as of a $U A S-M a d m$ construct was sufficient to rescue the lethality of the seven alleles and one copy of the genomic Madm construct fully reverted the pinhead phenotype (Materials and methods; Figure 3d), proving that Madm mutations caused the growth deficit.

\section{Allelic series of the EMS-induced Madm mutations}

To characterize the Madm growth phenotype more closely, we first attempted to order the Madm alleles according to their strength. To determine the lethal phase of the recessive lethal Madm EMS-alleles, they were combined with a deficiency $(D f(3 R)$ Exel7283) uncovering the Madm locus (see also Materials and methods). Development of mutant larvae ceased mostly in the third larval instar and in the prepupal stage. The onset of the prepupal stage was delayed by 2 to 10 days. Alleles 2D2, $2 U 3$, and $3 G 5$ led to strong growth deficits, most apparent in L3 larvae, whereas alleles $3 Y 2,4 S 3$, and $7 L 2$ caused almost no reduction in larval size. The allele 3T4 turned out to be a hypomorphic allele capable of producing few adult flies (less than $10 \%$ of the expected Mendelian ratio). 3T4 is caused by a point mutation leading to a premature translational stop (Figure 3e). However, it has been reported that the translation machinery can use alternative start codons in human Madm that are located further downstream [39]. Alternative start codons are also present in Drosophila Madm and may account for the hypomorphic nature of the allele $3 T 4$. 
As a second measurement of the strength of the Madm alleles, the severity of the pinhead phenotypes was judged. Consistent with the first assay, alleles $2 D 2,2 U 3$, and $3 G 5$ produced the most severe pinhead phenotypes (Figure 3c); alleles 3Y2, 4S3, and 7L2 displayed pinhead phenotypes of intermediate strength (Figure $3 \mathrm{~b}$ ); and allele $3 T 4$ led to a very mild reduction in head and eye size in the eyFLP/FRT assay (data not shown).

\section{Like BunA, Madm regulates cell number and cell size}

We further characterized the Madm growth phenotype by testing effects on cell number and cell size. To assess cell number, ommatidia were counted in scanning electron microscope (SEM) pictures taken of mosaic eyes largely homozygous mutant for Madm. Compared to control mosaic eyes (Figure 4a), Madm mutant eyes (Figure 4b,c) had significantly fewer ommatidia (Figure 4d). To detect changes in cell size, we determined the size of rhabdomeres - the light-sensing organelles of the photoreceptors - in tangential eye sections containing homozygous mutant clones (Figure 4a'-c'). In addition, we measured the entire cell bodies of photoreceptor cells. Madm mutant rhabdomeres and photoreceptor cell bodies were smaller than the controls (by 29-56\%; Figure $4 \mathrm{e}$, and data not shown). The reduction was cellautonomous because only homozygous mutant photoreceptor cells (marked by the absence of pigmentation) were affected.

Furthermore, the body size of rare hypomorphic mutant flies (produced with allele 3T4) was reduced (Figure 4f), and females were almost $40 \%$ lighter than controls (Figure 4g). Madm escapers also displayed malformations such as eye and wing defects. Eye sections revealed rotation defects, missing and extra photoreceptors, fused ommatidia, and cell-fate transformations (Figure $4 \mathrm{~h}$, and data not shown). Similar patterning defects were observed in Madm mutant clones in the eye (Figure 4b','). The wing phenotypes ranged from no defects to wing notches and an incomplete wing vein $\mathrm{V}$ (Figure 4i). All the growth and patterning defects of Madm mutant viable flies were reverted by a genomic rescue construct (Figure 4f,g; data not shown).

Thus, Madm controls cell number and cell size and also controls patterning processes in the eye and the wing. These phenotypes strongly resemble phenotypes displayed by bunA mutant cells and flies [12] [see Additional file 5 for wing notches], although the pinhead phenotype and the eye-patterning defects caused by the strong Madm alleles $2 D 2$ and $3 G 5$ are more severe.

\section{Madm and BunA cooperate to enhance growth}

$M a d m$ is a growth-promoting gene producing phenotypes reminiscent of bunA phenotypes and its gene product physically interacts with BunA. It is thus conceivable that the two proteins participate in the same complex to enhance growth. We tested for dominant genetic interactions between Madm and bunA in vivo. However, we did not detect dominant interactions in hypomorphic mutant tissues or flies (data not shown). Thus, we hypothesized that Madm and BunA form a molecular complex and, as a consequence, the phenotype of the limiting complex component is displayed. This hypothesis also implies that overexpression of Madm or BunA alone would not be sufficient to enhance the activity of the complex. As previously reported, overexpression of bunA from a $U A S-$ bunA construct did not produce any overgrowth phenotypes, unless co-overexpressed with $d S 6 K$ in a sensitized system in the wing [12] (Figure 5b,g). Similarly, with a UAS-Madm transgenic line, no obvious overgrowth phenotypes were observed (Figure 5c,h; Madm overexpression caused patterning defects, Materials and methods). However, co-overexpression of bunA and Madm by means of GMR-Gal4 resulted in larger eyes due to larger ommatidia (Figure $5 \mathrm{~d}$,e). Consistently, co-overexpression of UAS-Madm together with UAS-bunA using a wing driver (C10-Gal4) caused an overgrowth phenotype in the wing (Figure $5 \mathrm{i}, \mathrm{j}$ ). We observed additional tissue between the wing veins, resulting in crinkled wings. Thus, Madm and BunA cooperate to increase organ growth when overexpressed during eye and wing development.

\section{Discussion}

In the present study, we provide genetic evidence for an evolutionarily conserved function of the long TSC22DF isoforms in the control of cell and organ size. Because the long TSC22DF proteins share two conserved motifs in their amino-terminal parts, we set out to identify specific binding partners that cooperate with the long isoforms to promote cellular growth. The combination of AP-MS experiments with a genetic screen for novel mutations affecting growth [41] resulted in the identification of Madm as a strong candidate for such an interactor, illustrating the synergistic forces of the two approaches.

\section{The long TSC22DF proteins promote growth in Drosophila via an interaction with Madm}

We found that all long - but none of the short - members of the human TSC22DF are able to replace the function of BunA in the fly. Thus, the potential of long isoforms to positively regulate growth has been conserved through evolution. Conceivably, the various long isoforms present in mammals can, at least to some extent, substitute for one another and hence act in a (partially) redundant manner. However, our rescue experiments in Drosophila only demonstrate the potential of the long human TSC22DF proteins and do not allow us to draw any conclusions about their endogenous function. Whether 


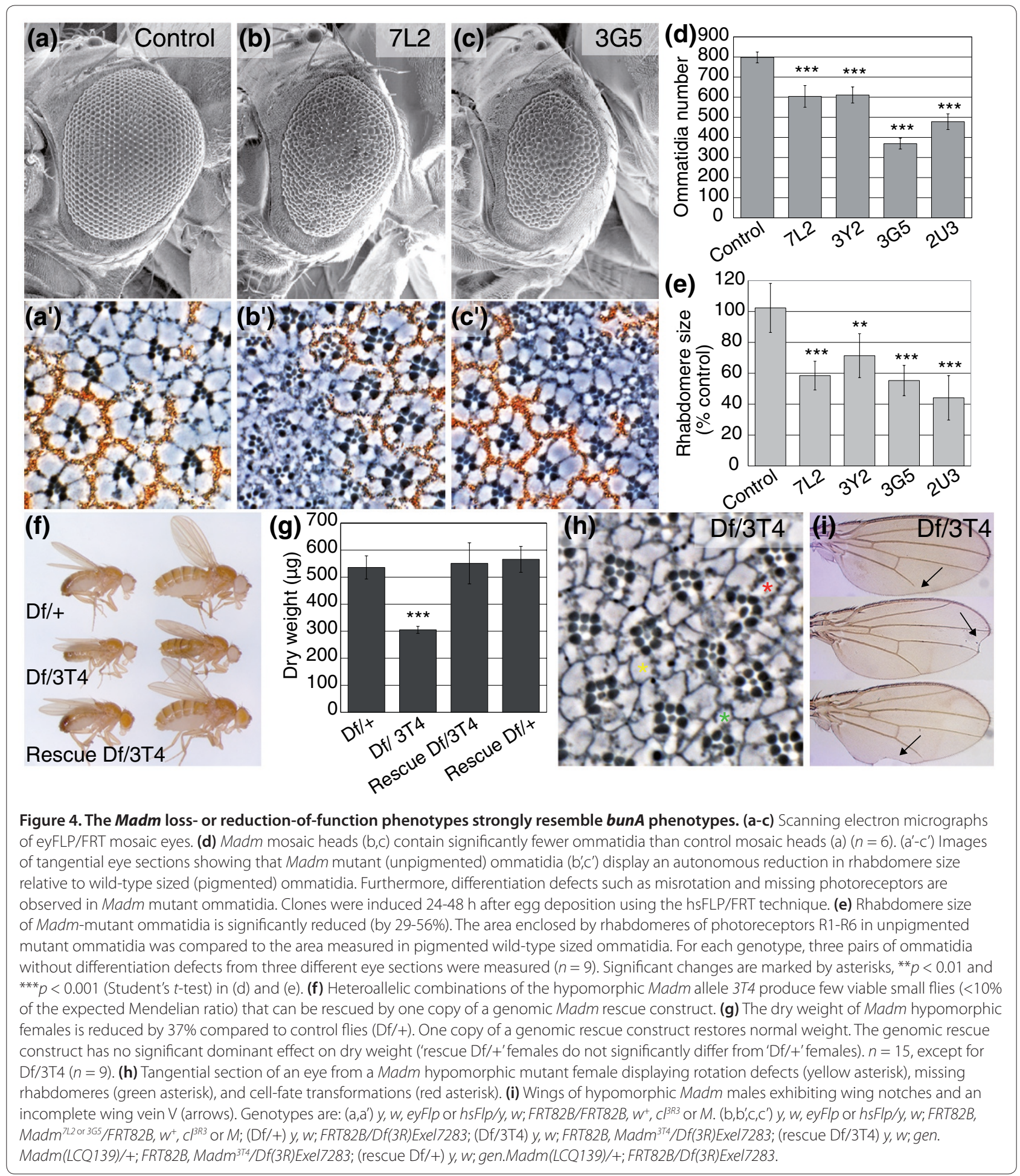

TSC22D1.1 is indeed a functional homolog of BunA in growth regulation and whether the short TSC22D1.2 protein antagonizes it need to be addressed in mammalian in vivo systems.

The potential of long human TSC22DF proteins to replace BunA function is likely to reside in conserved sequences shared by all long TSC22DF members. Alignments with long TSC22DF proteins revealed two short stretches of high conservation [12,13]. Intriguingly, two EMS-induced mutations leading to amino acid substitutions in the second conserved motif were isolated in a genetic screen for mutations affecting growth [12]. 


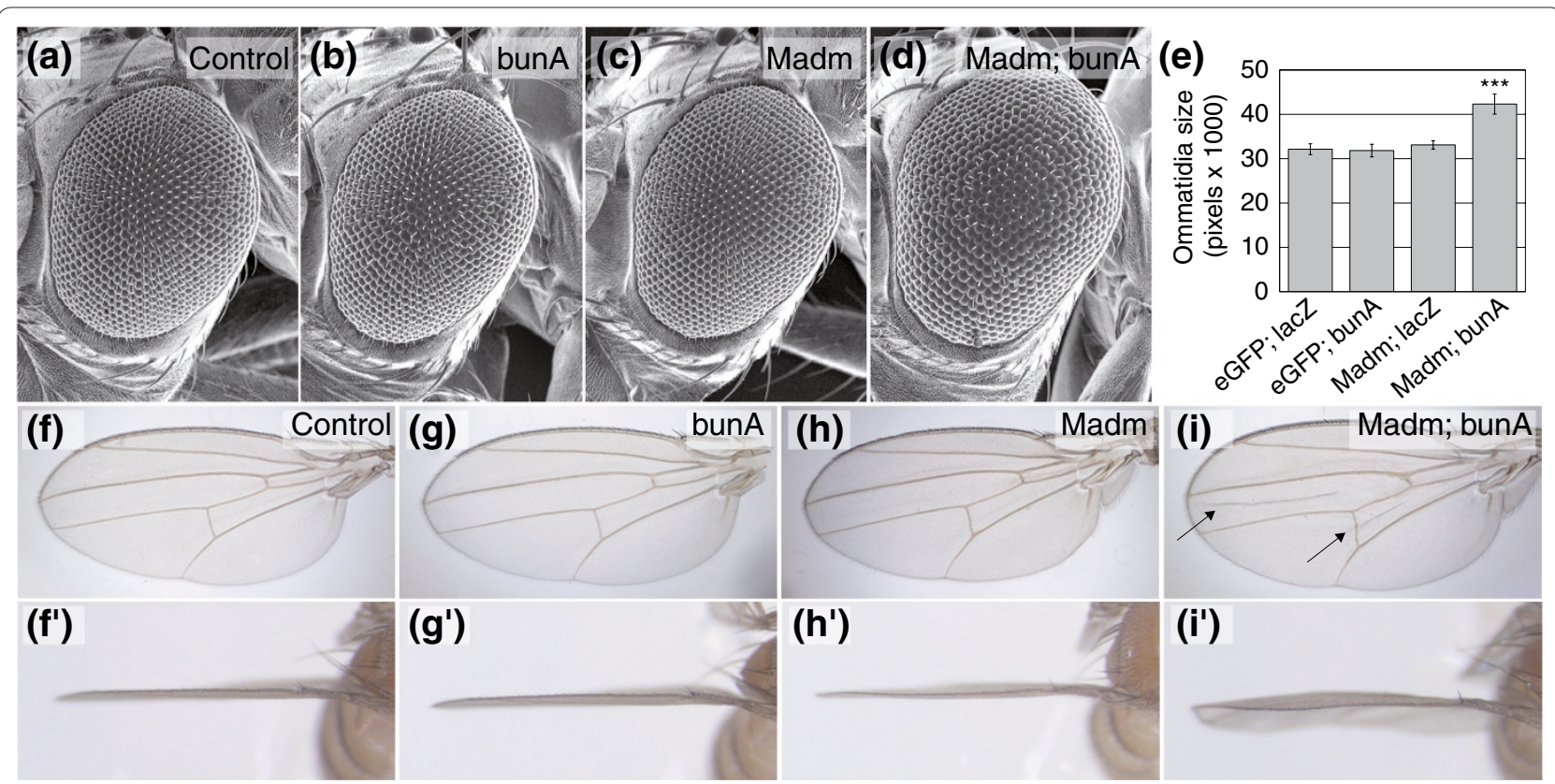

Figure 5. Co-overexpression of Madm and bunA causes overgrowth. (a-d) Scanning electron micrographs of adult eyes as a readout for the consequences of overexpression of bunA and Madm under the control of the GMR-Gal4 driver line late during eye development. Whereas expression of (b) bunA or (c) Madm singly does not cause a size alteration compared to the control (a), overexpression of both leads to increased eye size (d). (e) The size increase on bunA and Madm coexpression is due to larger ommatidia (Student's $t$-test, $n=9$, ${ }^{* * *} p<0.001$ ). (f-i) The growthpromoting effect of bunA and Madm co-overexpression is also observed in the wing. Single expression of either $\left(g, g^{\prime}\right)$ bunA or ( $\left.h, h^{\prime}\right)$ Madm during wing development (by means of (10-Ga/4) does not change wing size or curvature visibly. However, their combined expression causes a slight overgrowth of the tissue between the wing veins, resulting in a wavy wing surface and wing bending $\left(\mathrm{i}^{\prime}\right)$, manifested as folds between wing veins in (i) (arrows). Genotypes are: (a) y, w; GMR-Gal4/UAS-eGFP; UAS-lacZ/+; (b) y, w; GMR-Gal4/UAS-eGFP; UAS-bunA/+; (c) y, w; GMR-Gal4/UAS-Madm; UAS-lacZ/t; (d) y, w; GMR-Gal4/UAS-Madm; UAS-bunA/t; (f) y, w; UAS-eGFP/t;C10-Gal4/UAS-lacZ; (g) y, w; UAS-eGFP/t;C10-Gal4/UAS-bunA; (h) y, w; UAS-Madm/+;C10-Gal4/UAS-lacZ; (i) y, w; UAS-Madm/+;C10-Gal4/UAS-bunA.

The corresponding alleles behaved as strong bunA hypomorphs that were recessive lethal and caused severe growth deficits. BunA binds via the second conserved motif to Madm and at least one mutation weakens the binding but does not abolish it. As the motif 2 is present in all long TSC22DF isoforms, it is likely that all of them can bind Madm. In fact, the long human isoform TSC22D4 is able to do so, as uncovered in a large-scale $\mathrm{Y} 2 \mathrm{H}$ study $[42,43]$. So far, we could not assign any function to the first conserved motif. Because this motif is heavily phosphorylated [44], we speculate that it is important for the regulation of BunA activity.

Because short isoforms can heterodimerize with long isoforms, as reported for TSC-22 (TSC22D1.2) and TSC22D4 [13], they may interact indirectly with Madm. This could explain why human Madm was found to interact with the bait protein TSC-22 (TSC22D1.2) in a high-throughput analysis of protein-protein interactions by immunoprecipitation followed by mass spectrometry (IP/MS) [43,45]. Moreover, we found that the short isoform BunB interacts with Drosophila Madm in a co-IP but not in a $\mathrm{Y} 2 \mathrm{H}$ assay. Heterodimers of BunA and short Bun isoforms exist in Drosophila S2 cells because we found that a small fraction of endogenous BunA did co-immunoprecipitate with tagged BunB and BunC versions (data not shown). However, we failed to identify short Bun isoforms as BunA heterodimerization partners in the AP-MS experiments. One possible explanation is that the peptides specific for short Bun isoforms are very low abundant. This might also explain why they were not detected when a catalog of the Drosophila proteome was generated [46].

In mammalian cells, both IP/MS and Y2H experiments provided evidence for a physical interaction between Madm and TSC22DF proteins [42,43]. Our study extends these findings in two ways. We demonstrate that only long TSC22DF proteins directly bind to Madm, and we also provide evidence for the biological significance of this interaction in growth control.

\section{Biological functions of Madm}

Madm has been implicated in ER-to-Golgi trafficking because overexpression of Madm affected the intracellular transport of a Golgi-associated marker in COS-1 cells [47]. In addition, Madm localizes to the nucleus, the cytoplasm and Golgi membranes in Drosophila, and an 
RNA interference (RNAi)-mediated knockdown of $M a d m$ in cultured cells interfered with constitutive protein secretion [46,48]. In Xenopus, Madm is important for eye development and differentiation [49]. Thus, it is apparent that Madm is involved in biological processes other than growth control. As a consequence, disruption of Madm leads to complex phenotypes partly different from bunA phenotypes, and concomitant loss of Madm and $\operatorname{bun} A$ causes an even stronger growth decrease than the single mutants [see Additional file 5]. In addition to the Madm growth phenotypes, we observed patterning defects, for example in the adult fly eye and wing. Similar phenotypes were detected when bunA function was absent or diminished [12], yet the patterning defects caused by Madm and the Madm pinhead phenotype appeared to be more pronounced. Alternatively, these more pronounced phenotypes could arise from a lower protein stability of Madm compared with BunA, leading to more severe phenotypes in the eyFLP/FRT assay. However, in contrast to the effects of BunA overexpression, the overexpression of Madm early during eye and wing development led to severe differentiation defects. These phenotypes could be caused by Madm-interaction partners other than BunA that function in different biological processes.

Madm is an adapter molecule that has several interaction partners in mammals. Originally, it was proposed that Madm - also named nuclear receptor binding protein 1 (NRBP1) in humans - binds to nuclear receptors because of the presence of two putative nuclear-receptorbinding motifs [39]. However, Madm has never been experimentally shown to bind to any nuclear receptor. Furthermore, the nuclear-receptor-binding motifs are not conserved in Drosophila. From studies in mammalian cells, it is known that Madm can bind to murine Mlf1 [40], Jab1 (Jun activation domain-binding protein 1) [50], activated Rac3 (Ras-related C3 botulinum toxin substrate 3) [47], Elongin B [51], and the host cellular protein NS3 of dengue virus type 2 [52]. Indeed, in our AP-MS experiment where HA-Madm was used as bait, we identified Elongin B but not Mlf1 (dMlf in Drosophila), Jab1 (CSN5 in Drosophila) or Rac3 (RhoL in Drosophila). It is possible that these interactions are not very prominent or even absent in Drosophila S2 cells.

\section{The Madm-BunA growth-promoting complex}

Madm and BunA are limiting components of a newly identified growth-promoting complex because genetic disruptions of bunA and Madm both result in a reduction in cell number and cell size. However, to enhance the activity of the complex and thereby to augment organ growth, simultaneous overexpression of both components is required. In the reduction-of-function situation, we did not detect genetic interactions between bunA and
Madm. Thus, we hypothesize that both proteins are essential components of a growth-promoting complex. As a consequence, the phenotype of the limiting protein will be displayed no matter whether the levels of the other protein are normal or lowered.

It is not clear whether additional proteins are part of the Madm-BunA growth-regulating complex. Heterodimerization partners of BunA or other Madm-binding proteins are candidate complex members. Conversely, Madm-binding partners could form distinct complexes mediating different functions. These complexes may negatively regulate each other by competing for their shared interaction partner Madm. Indeed, we observed a suppressive effect when dMlf or CSN5 were co-overexpressed along with Madm and BunA in the developing eye (data not shown). Thus, other Madm-binding partners will directly or indirectly influence the growthpromoting function of the Madm-BunA complex.

We found that GFP-BunA co-localizes with the Golgi marker GMAP210 in Drosophila S2 cells. Interestingly, it has been suggested that mammalian as well as Drosophila Madm plays a role in ER-to-Golgi transport, and it has been reported that Madm localizes to the cytoplasm, weakly to the nucleus, and to the Golgi in Drosophila S2 cells [48]. We observed a similar subcellular localization of both HA-Madm and HA-Madm(R525H) when expressed at low levels (data not shown). The Golgi localization was lost in cells expressing higher levels of HA-Madm, possibly because the cytoplasm was loaded with protein. Intriguingly, the Golgi localization of HA-Madm, but not of HA-Madm(R525H), was completely restored in cells coexpressing GFP-BunA and HA-Madm at relatively high levels. Thus, BunA is able to direct Madm to the Golgi, and the Golgi may be the site of action of the Madm-BunA growth-regulating complex. However, because our investigation was restricted to overexpression studies, the subcellular localization of endogenous Madm and BunA remains to be analyzed.

How could binding of Madm modulate the function of BunA? Madm could have an impact on the stability, the activity or the subcellular localization of BunA. We analyzed the amount of endogenous and overexpressed BunA protein in cultured Drosophila cells with diminished or elevated Madm levels, produced by RNAi with double-stranded RNA (dsRNA) or by overexpression, respectively, but did not observe any effect (data not shown). Thus, Madm does not fundamentally affect the stability of BunA. The putative transcription factor BunA localizes to the cytoplasmic and not to the nuclear fractions in Drosophila [31,46]. Because Madm possesses NES and NLS sequences, it is likely to shuttle between the cytoplasm and the nucleus [52] and it might therefore transport BunA to the nucleus, where BunA could act as a transcription factor. So far, however, we have not 
detected nuclear translocation of BunA (data not shown). The activity of BunA could be controlled by phosphorylation events, as it has been described for numerous transcription factors. An attractive model is that a kinase binding to Madm phosphorylates BunA. An analogous model was proposed for murine Mlf1 as Madm binds to an unknown kinase that phosphorylates Madm itself and a 14-3-3zeta-binding site in Mlf1, possibly resulting in 14-3-3-mediated sequestration of Mlf1 in the cytoplasm [40].

Further studies will be required to solve the exact mechanism by which Madm and BunA team up to control growth. We anticipate that our findings will encourage studies in mammalian systems on the function of long TSC22DF members, in particular TSC22D1.1, in growth control.

\section{Conclusions}

The mechanism by which the tumor suppressor TSC-22 acts has remained unclear, and the functional analysis of TSC-22 is hampered because of redundancy and various possible interactions among the homologous TSC22DF proteins. In a previous study, we showed that the Drosophila long class TSC22DF isoforms are positive growth regulators. Here, we report that the long human TSC22DF isoforms are able to substitute for BunA function when expressed in the fly. To illuminate the mechanism by which long TSC22DF isoforms promote growth, we searched for BunA binding partners. A combined proteomic and genetic analysis identified the adapter protein Madm. Drosophila Madm is a positive growth regulator that increases organ growth when co-overexpressed with BunA. We propose that the BunAMadm growth-promoting complex is functionally conserved from flies to humans.

\section{Materials and methods \\ Breeding conditions and fly stocks}

Flies were kept at $25^{\circ} \mathrm{C}$ on food described in [53]. For the rescue experiment bun ${ }^{200 B}$ [12], UAS-bunA [31], armGal4, da-Gal4, and Act5C-Gal4 (Bloomington Drosophila Stock Center), and vas- $\phi C 31-z h 2 A ; Z H$-attP-86Fb [35] flies were used. For the genetic mosaic screen, $y, w$, eyFLP; FRT82B, $\mathrm{w}^{+}, \mathrm{cl}^{3 R 3} / \mathrm{TM} 6 \mathrm{~B}, \mathrm{~Tb}, \mathrm{Hu}$ flies [54] were used. Clonal analyses in adult eyes were carried out with $y, w, h s F L P ; F R T 82 B, w^{+}, M / T M 6 B, T b, H u, y^{+}$. For rescue experiments, allelic series, and the analysis of hypomorphic mutant Madm flies, Df(3R)Exel7283 (Bloomington Drosophila Stock Center) was used. In hypomorphic bunA flies displaying wing notches, the alleles $b u n^{A-P 519 L}$ [12] and bun ${ }^{r 043}$ [31] were combined. Madm, bunA double-mutant mosaic heads were generated with $y, w$, eyFLP; FRT40A, $w^{+}, \mathrm{cl}^{2 L 3} / C y O ; F R T 82 B, w^{+}, \mathrm{cl}^{3 R 3} / T M 6 B$, $\mathrm{Tb}, \mathrm{Hu}$ [54] flies, bun allele $A-Q 578 X$ [12], the UAS hairpin line 19679 (RNAi bun) [55], and ey-Gal4 [56]. The overexpression studies in the eye and wing were done with GMR-Gal4 [57] and C10-Gal4 [58], UAS-eGFP, and UAS-lacZ (Bloomington Drosophila Stock Center).

\section{Generation of transgenic flies}

bunA cDNA was subcloned from a UAS-bunA plasmid [31] into the pUAST-attB vector [35] using EcoRI sites. The bunA ORF was PCR-amplified from a UAS-bunA plasmid [31], cloned into the pENTR-D/TOPO vector (Invitrogen) and subcloned into a Gateway-compatible pUAST-attB vector (J Bischof, Institute of Molecular Biology, University of Zurich; unpublished work) by clonase reaction (LR clonase II enzyme).

The human ORFs TSC22D1.1, TSC22D1.2, TSC22D3v1-3 and TSC22D4 were derived from the cDNA of a normal prostate tissue sample. This sample was derived from a radical prostatectomy specimen at the Department of Urology, University of Berne as described previously [4]. The ORF TSC22D2 was derived from the pOTB7 vector carrying the TSC22D2 full-length cDNA (Open Biosystems, clone ID 5454441). ORFs were PCR-amplified, cloned into the pGEM-T Easy vector (Promega) and subsequently cloned into the pcDNA3.1/Hygro(+) vector (Invitrogen). The ORFs TSC22D1.1 and TSC22D2 were subcloned from pGEM-T Easy to pUAST-attB using EcoRI. The ORF TSC22D1.2 was subcloned from pcDNA3.1/Hygro(+) to the pBluescript II $\mathrm{KS}(+/-)$ vector using HindIII and XhoI, then further subcloned into the pUAST vector [34] using EcoRI and Xhol, and finally cloned into the pUAST-attB vector with EcoRI and XbaI. The ORFs TSC22D3v1-3 and TSC22D4 were PCRamplified from cDNA-containing pGEM-T Easy plasmids and cloned into pUAST-attB using EcoRI and NotI (restriction sites added by PCR). The pUAST-attB plasmids were injected into vas- $\phi C 31-z h 2 A ; Z H$-attP-86Fb embryos [35].

Madm cDNA was cleaved by EcoRI and HindIII double digestion from expressed sequence tag (EST) clone LD28567 (Berkeley Drosophila Genome Project) and subcloned into pUAST using the same restriction sites to generate the UAS-Madm construct. Madm genomic DNA (from 559 bp upstream of $M a d m$ exon 1 (containing exon 1 of the neighboring gene CG2097) to 1,681 bp downstream of Madm exon 2) was amplified by PCR using forward primer GCTCTAGAAGGCGATGCGATGACCAGCTC and reverse primer GAGATCTTCATGACGTTTTCCGCGCACTCGAGT. The PCR product was digested with BglII and XbaI and subcloned into the transformation vector $\mathrm{pCaspeR}$.

\section{Gateway cloning for Drosophila cell culture and yeast two-hybrid assays}

The complete and partial ORFs of bunA and Madm were PCR-amplified from a $p U A S T-b u n A[31]$ and a UAS-Madm 
plasmid, respectively, and cloned into the pENTR/DTOPO vector. The point mutations in $p E N T R-D / T O P O-$ bunA and $-M a d m$ were introduced by substitution of a BamHI/DraI and an FspI/SacI fragment that was PCRamplified using mutated primers. By clonase reaction (LR clonase II) the inserts were transferred to the following destination vectors: pMT-HHW-Blast (O Rinner, IMSB, ETH Zurich, unpublished work), pMT-GW-Blast, pDEST22, and pDEST32. Additionally, the GFP ORF was cloned into the pMT-HHW-Blast vector, leading to the production of HA-tagged GFP as a negative control for co-IP experiments. The pMT-HHW-Blast vector is based on the pMT-V5HisA vector (Invitrogen) carrying a metallothionein-inducible promoter. The multiple cloning site and tag sequences were replaced by the Gateway cassette, including the coding sequence for a triple HA-tag from the pAHW destination vector (Invitrogen). The blasticidin-resistance cassette was cloned from the pCoBlast vector (Invitrogen) into the pMT-V5HisA vector backbone. The pMT-HHW-Blast vector was modified by exchanging an AgeI/EcoRI fragment containing the GFP coding region derived from the pAGW destination vector.

\section{Cell culture conditions and cell transfections}

Drosophila embryonic S2 cells were grown at $25^{\circ} \mathrm{C}$ in Schneider's Drosophila medium (Gibco/Invitrogen) supplemented with $10 \%$ heat-inactivated fetal calf serum (FCS), as well as penicillin and streptomycin. S2 cells were transfected according to the Effectene transfection protocol for adherent cells (Qiagen). To generate stable cell lines, transfected S2 cells were selected for 14-30 days in Schneider's medium containing $25 \mu \mathrm{g} / \mathrm{ml}$ blasticidin and afterwards propagated in Schneider's medium containing $10 \mu \mathrm{g} / \mathrm{ml}$ blasticidin.

\section{Pulldown experiments analyzed by LC-MS/MS}

Before affinity purification, Drosophila S2 cells were grown in shaking flasks. Bait expression was induced by $600 \mu \mathrm{M} \mathrm{CuSO}_{4}$ for at least $16 \mathrm{~h}$. For affinity purification the cell pellets were lysed on ice for 30 minutes in $10 \mathrm{ml}$ HNN (50 mM HEPES pH 7.5, 5 mM EDTA, $250 \mathrm{mM}$ $\mathrm{NaCl}, 0.5 \%$ NP40, $1 \mathrm{mM}$ PMSF, $50 \mathrm{mM} \mathrm{NaF}, 1.5 \mathrm{mM}$ $\mathrm{Na}_{3} \mathrm{VO}_{4}$, protease inhibitor cocktail (Roche)) in the presence of $3 \mathrm{mM}$ dithiobis-(succinimidyl propionate) (DSP) with ten strokes using a tight-fitting Dounce homogenizer. Reactive DSP was quenched by adding $1 \mathrm{ml}$ Tris $\mathrm{pH}$ 7.5. Insoluble material was removed by centrifugation and the supernatant was precleared using $100 \mu \mathrm{l}$ Protein A-Sepharose (Sigma) for $1 \mathrm{~h}$ at $4^{\circ} \mathrm{C}$ on a rotating shaker. After removal of the Protein A-Sepharose, $100 \mu \mathrm{l}$ Agarose anti-GFP beads (MB-0732) or Agarose monoclonal mouse anti-HA beads (Sigma A2095) were added to the extracts and incubated for $4 \mathrm{~h}$ at $4^{\circ} \mathrm{C}$ on a rotating shaker. Immunoprecipitates were washed four times with 20 bed volumes of lysis buffer and three times with 20 bed volumes of buffer without detergent and protease inhibitor. The proteins were released from the beads by adding three times $150 \mu \mathrm{l} 0.2 \mathrm{M}$ glycine $\mathrm{pH} 2.5$. Following neutralization with $100 \mu \mathrm{l} 1 \mathrm{M} \mathrm{NH}_{4} \mathrm{CO}_{3}$, the eluates were treated with $5 \mathrm{mM}$ tris(2-carboxyethyl)phosphine (TCEP) to reduce $\mathrm{S}-\mathrm{S}$ bonds and DSP crosslinkers for 30 minutes at $37^{\circ} \mathrm{C}$, and alkylated with $10 \mathrm{mM}$ iodoacetamide for 30 minutes at room temperature in the dark. For tryptic digestion, $1 \mu \mathrm{g}$ trypsin was added to the eluate and incubated at $37^{\circ} \mathrm{C}$ overnight.

Nanoflow-LC-MS/MS was performed by coupling an UltiMate HPLC system (LC-Packings/Dionex) in-line with a Probot (LC-Packings/Dionex) autosampler system and an LTQ ion trap (Thermo Electron). Samples were automatically injected into a $10-\mu \mathrm{l}$ sample-loop and loaded onto an analytical column $(9 \mathrm{~cm} \times 75 \mu \mathrm{m}$; packed with Magic C18 AQ beads $5 \mu \mathrm{m}, 100 \AA$ (Michrom BioResources)). Peptide mixtures were delivered to the analytical column at a flow rate of $300 \mathrm{nl} /$ minute of buffer A (5\% acetonitrile, $0.2 \%$ formic acid) for 25 minutes and then eluted using a gradient of acetonitrile (10-45\%; $0.5 \% /$ minutes) in $0.2 \%$ formic acid. Peptide ions were detected in a survey scan from 400 to 2,000 atomic mass units (amu; one to two $\mu$ scans) followed by three to six data-dependent MS/MS scans (three $\mu$ scans each, isolation width $2 \mathrm{amu}$, dynamic exclusion list 250, dynamic exclusion time $240 \mathrm{sec}$, two repeats) in an automated fashion. Following analysis, raw MS/MS data were converted to Mascot generic format (MGF) files that were used to identify the corresponding peptides using the Mascot database search tool (MatrixScience) $[59,60]$. MS/MS spectra were searched against the Drosophila protein database (dmel_r5.18_FB2009_05 released 20090529) [60] with the following criteria: requirement for trypsin digestion; peptide mass tolerance $2 \mathrm{Da}$; fragment mass tolerance 0.6 Da; missed cleavage for trypsin 3; fixed modification carbamidomethyl (C); variable modifications acetyl (N-term), oxidation (M), CAM-thiopropanoyl (K) (derived from DSP crosslinker). All peptide assignments with Mascot ion score larger than the homology score and with Mascot expect score smaller than 0.05 (significant ion score) were considered as good hits.

\section{Co-localization studies}

For immunofluorescence Drosophila S2 cells were stimulated for $4 \mathrm{~h}$ with $600 \mu \mathrm{M} \mathrm{CuSO}_{4}$ and spread on concanavalin-A-treated slides and fixed with $4 \%$ paraformaldehyde. Mouse anti-HA (1:200, Roche 166606), rabbit anti-GMAP210 (1:200) [38], goat anti-mouse Cy3 (1:200, Amersham PA43002) and goat anti-rabbit Cy3 (1:200, Amersham PA43004) antibodies were used. 
Nuclei were visualized by DAPI $(100 \mathrm{ng} / \mathrm{ml})$ staining. For imaging, a Leica TCS SPE confocal microscope was used.

Statistical analysis of the dependence of HA-Madm localization on GFP-BunA was carried out in S2 cells transiently transfected two days before analysis. Cells were co-transfected with $p M T-H A-M a d m$ or $p M T-H A$ $\operatorname{Madm}(R 525 H)$ and with either empty pMT-GW-Blast (for control GFP expression) or with pMT-GFP-bunA. Fifty cells were analyzed for each combination. The expressed HA-Madm and HA-Madm(R525H) proteins showed a similar diffuse localization in the cytoplasm of cells strongly expressing GFP (50 out of 50 each). By contrast, HA-Madm localized to dots (punctae) in cells displaying a readily detectable GFP-BunA signal (50 out of 50). In 17 out of 50 cells, GFP-BunA directed HA-Madm(R525H) localization in punctae.

\section{GPF-tagged versions of BunA and Madm}

To generate flies expressing amino-terminally GFPtagged versions of BunA and Madm, the respective ORFs were subcloned by clonase reaction from pENTR/DTOPO to pUAST-GW-AttB (the Gateway-compatible pUAST-AttB vector was provided by I Pörnbacher, IMSB, ETH Zurich; unpublished work). The plasmid containing bunA was injected into vas- $\phi C 31-z h 2 A ; Z H$-attP-86Fb embryos [35], and the Madm-containing plasmid into vas- $\phi C 31-z h 2 A ; Z H$-attP-51D embryos [35]. Leaky expression of $U A S-G F P$-bunA was sufficient to completely rescue the lethality of $b_{u n}{ }^{200 B / 200 B}$ mutants. Expression of UAS-GFP-Madm under the control of the arm-Gal4 driver line resulted in a rescue (approximately $50 \%$ of the expected Mendelian ratio) of the lethality of $\mathrm{Madm}^{3 \mathrm{X}_{2}}$ / Df(3R)Exel7283 animals.

\section{Co-immunoprecipitation and western blotting}

Stable Drosophila S2 cell lines capable of producing GFP (negative control) or GFP-tagged Bun versions were transiently transfected with $H A-M a d m$ plasmids. Two days after transient transfection, confluent Drosophila S2 cells ( $2.3 \mathrm{ml}$ corresponding to one well of a 6-well-plate) were induced for $4 \mathrm{~h}$ with $\mathrm{CuSO}_{4}(600 \mu \mathrm{M})$, harvested, and washed with PBS. Cells were lysed in IP buffer (120 $\mathrm{mM} \mathrm{NaCl}, 50 \mathrm{mM}$ Tris $\mathrm{pH}$ 7.5, $20 \mathrm{mM} \mathrm{NaF}, 1 \mathrm{mM}$ benzamidine, $1 \mathrm{mM}$ EDTA, $6 \mathrm{mM}$ EGTA, $15 \mathrm{mM}$ $\mathrm{Na}_{4} \mathrm{P}_{2} \mathrm{O}_{7}, 0.5 \%$ Nonidet P-40, 1x Complete Mini Protease Inihibitor (Roche)). After incubation for 15 minutes on an orbital shaker at $4^{\circ} \mathrm{C}$, solubilized material was recovered by centrifugation at 13,000 rpm for 15 minutes and supernatants were collected. Cell lysates were incubated for $4 \mathrm{~h}$ at $4^{\circ} \mathrm{C}$ under rotation either with $20 \mu \mathrm{l}$ Agarose anti-GFP beads (MB-0732) or Agarose monoclonal mouse anti-HA beads (Sigma A2095) that had been equilibrated with IP buffer. Beads were then washed five times with IP buffer and boiled for 5 minutes at $95^{\circ} \mathrm{C}$ in Laemmli buffer. Proteins contained in the supernatant were separated by SDS-PAGE. For western blotting, the Hybond ECL Nitrocellulose membrane (Amersham), the ECL detection reagent (Amersham), and the following antibodies were used: rabbit anti-Bun $(1: 1,000)$ [33], rabbit anti-BunA (1:2,000), mouse anti-HA (1:3,000, Roche 166606), monoclonal mouse anti-GFP (1:1,000, Roche 11 814460 001), monoclonal mouse anti-rabbit horseradish peroxidase (HRP) $(1: 10,000$, Jackson Lab), sheep antimouse HRP (1:10,000, Amersham NA931).

The rabbit anti-BunA antibody was generated using the following peptide to immunize rabbits: $\mathrm{H}_{2} \mathrm{~N}$ TNRKPKTTSSFEC-CONH ${ }_{2}$ (Eurogentec). The serum was double-affinity purified against the peptide coupled to a column. Specificity of the antibody was shown by western blotting: Drosophila S2 cells were incubated for 2 days with dsRNA [61] that targets either the ORF of bunA (500 bp long dsRNA) or of GFP as a control (700 bp long dsRNA). Primer sequences are available on request.

\section{Yeast two-hybrid experiments}

For the $\mathrm{Y} 2 \mathrm{H}$ experiments the Gateway-compatible ProQuest Two-Hybrid System (Invitrogen) including the pDEST32 and pDEST22 vectors was used. Transformation of a MaV203 strain was achieved with the LiAc/ SS carrier DNA/polyethylene glycol (PEG) method. To identify interactions, yeast cells were tested for HIS3 reporter gene expression under the control of the HIS promoter. The competitive inhibitor 3-amino-1,2,4triazole (3-AT) was used to dampen leaky expression. The 3-AT concentration needed to restrain leakiness was determined to be $10 \mathrm{mM}$ by almost suppressing the growth of negative control strains that have been transformed with an empty pDEST22 and/or pDEST32 vector (every combination of negative controls was examined). For each interaction to be tested, five yeast colonies were pooled and grown overnight in -Leu -Trp medium. Seven microliters of dilutions of $\mathrm{OD}_{600}=0.1$, 0.01 , and 0.001 with double-distilled $\mathrm{H}_{2} \mathrm{O}$ were spotted on -Leu -Trp -His +3-AT (10 mM) plates and incubated for $52-56 \mathrm{~h}$ at $30^{\circ} \mathrm{C}$. As a control for a correct dilution series they were also spotted on -Leu -Trp -His plates without 3-AT (data not shown).

\section{eyFLP/FRT screen, mapping of EMS mutations, and rescue experiments}

The eyFLP/FRT technique [54] was used to produce mosaic flies with eyes and head capsules largely homozygous for randomly induced mutations. The rest of the body (including the germline) remained heterozygous and was therefore phenotypically wild type (screen described in [41]). The seven EMS alleles of a complementation group on $3 \mathrm{R}$ were mapped using visible 
markers (P-elements) to the cytological region 83-84. With deletions that failed to complement the lethality of EMS alleles $(D f(3 R) E D 5196, \quad D f(3 R) E D 5197, D f(3 R)$ Exel7283, and $D f(3 R)$ Exel6145) the candidate region was narrowed down to the cytological interval 83C1-4. The EP-element EP3137 inserted in the 5' untranslated region of the Madm locus failed to complement the lethality of the EMS alleles.

The UAS/Gal4 system [34] was used to test whether ubiquitous expression of a UAS-Madm construct during development - achieved by the arm-Gal4, da-Gal4, and Act5C-Gal4 driver lines - rescued the recessive lethality of the EMS alleles. Using arm-Gal4, the viability of combinations of EMS alleles (2U3, 3G5, 3Y2, and 7L2) with the deletion $D f(3 R)$ Exel7283 was completely restored and the lethality of heteroallelic combinations among EMS alleles was partially rescued. One copy of a genomic Madm rescue construct (LCQ139) - comprising $559 \mathrm{bp}$ of the upstream region, the two Madm exons and the intron, and 1,681 bp of downstream sequences - was sufficient to entirely rescue the lethality of heteroallelic combinations of EMS alleles ( $2 U 3$ and $3 G 5)$ and of the hemizygous alleles (tested with the deficiency $D f(3 R)$ Exel7283). In addition, the pinhead phenotypes of the EMS alleles $2 U 3$ and $3 G 5$ were reverted by one copy of the genomic construct.

\section{Determination of allelic series}

Animals were reared on apple agar plates supplemented with yeast at $25^{\circ} \mathrm{C}$. Allelic series was determined by crossing Madm EMS alleles (y, w; FRT82B, Madm-) $T M 6 B, T b, H u)$ to a deficiency ( $y, w ; D f(3 R)$ Exel7283/ $T M 6 B, T b, H u)$ uncovering the entire $M a d m$ locus. In addition, the development of $M a d m^{2 U 3 / 3 G 5}$ and $M a d m^{3 Y 2 / 7 L 2}$ animals was compared with the development of $\mathrm{Madm}^{243}$ or $3 G 5 / D f(3 R)$ Exel7283 and $\mathrm{Madm}^{3 Y 2}$ or ${ }^{7 L 2} /$ Df(3R)Exel7283 animals, respectively. Heteroallelic combinations of the Madm EMS alleles, except for 3T4, resulted in larval phenotypes that were as strong as the phenotypes caused in the hemizygous condition.

\section{Analysis of adult flies}

Clones in the adult eyes were induced 24-48 $\mathrm{h}$ after egg deposition (AED) by a heat shock for $1 \mathrm{~h}$ at $34^{\circ} \mathrm{C}$ in animals of the genotype $y, w, h s F L P / y, w ; F R T 82 B, w^{+}$, $M / F R T 82 B, M a d m$. Adult fly heads were halved using a razor blade and stored up to $1 \mathrm{~h}$ in Ringer's solution on ice. Eyes were then fixed and processed as described in [62]. Pictures were taken with a Zeiss Axiophot microscope. In tangential eye sections, the areas enclosed by rhabdomeres and cell bodies from photoreceptors R1-R6 were measured in mutant ommatidia (lacking pigmentation) and in neighboring wild-type sized (pigmented) ommatidia using Adobe
Photoshop. Student's t-test was used to determine significance.

For the analysis of the ommatidia number in mosaic eyes and the analysis of ommatidia size in overexpression studies, flies were frozen at $-20^{\circ} \mathrm{C}$ before taking SEM pictures. All ommatidia were counted and the area of seven adjacent ommatidia (rosette) in the center was measured using Adobe Photoshop.

Freshly eclosed hypomorphic mutant Madm flies were kept on fresh food for 2 days. Flies were exposed to $95^{\circ} \mathrm{C}$ for 5 minutes and air-dried at room temperature for 3 days. The dry weight of individual flies was determined with a Mettler Toledo MX5 microbalance. Significance was assessed using Student's $t$-test.

Adult wings were mounted in Euparal after dehydration in $70 \%$ and subsequently $100 \%$ ethanol. Pictures were taken with a Zeiss Axiophot microscope. Significance was assessed using Student's $t$-test.

For the overexpression studies, a set of Gal4 driver lines was crossed to a UAS-Madm transgenic line. No obvious phenotypes were observed using arm-Gal4, da-Gal4, ppl-Gal4, and sev-Gal4. Ubiquitous overexpression achieved with Act5C-Gal4 resulted in lethality. Expression in the dorsal compartment of the developing wing (ap-Gal4) led to deformed wings. MS1096-Gal4 directed expression in the developing wing produced vein defects and blisters in the adult wing. Expression of Madm during early eye development (ey-Gal4) produced patterning defects (rough and small adult eyes) whereas Madm expression later during eye development (GMRGal4) produced growth phenotypes when co-overexpressed with UAS-bunA.

Additional file 1: Candidate list of three AP-MS experiments, including supporting information.

Additional file 2: Mapping of the BunA region sufficient for the interaction with Madm by means of $\mathrm{Y} 2 \mathrm{H}$ and co-IP experiments.

Additional file 3: Mapping of the Madm region sufficient for the interaction with BunA by means of $\mathrm{Y} 2 \mathrm{H}$ and co-IP experiments.

Additional file 4: Alignments of BunA and Madm interaction regions and western blots showing the specificity of the anti-BunA antibody.

Additional file 5: Pictures of wing notches in bunA hypomorphic flies and Madm, bunA double-mutant mosaic eyes.

\section{Abbreviations}

$\mathrm{AP}=$ affinity purification; $\mathrm{Bun}=$ Bunched $; \mathrm{cO}-\mathrm{IP}=$ co-immunoprecipitation; EMS = ethyl methanesulfonate; GFP = green fluorescent protein; $\mathrm{HA}=$

hemagglutinin; $\mathrm{LC}=$ liquid chromatography; Madm = Mlf1 adapter molecule; MGF = Mascot generic format; MIf1 = Myeloid leukemia factor $1 ; \mathrm{MS}=$ mass spectrometry; NES = nuclear export signal; NLS = nuclear localization signal; TSC-22 = Transforming growth factor- $\beta 1$ stimulated clone-22; TSC22DF $=$ TSC 22 domain family; $\mathrm{Y} 2 \mathrm{H}=$ yeast two-hybrid 


\section{Acknowledgements}

We thank Anni Straessle, Béla Brühlmann, Christof Hugentobler and Rita Bopp for technical assistance; Johannes Bischof, Oliver Rinner and Ingrid Pörnbacher for reagents; Fabian Rudolf and Daniel Bopp for help with the yeast two-hybrid experiments; Timo Glatter, Matthias Gstaiger, Cristian Köpfli and Sandra Götze for assistance in the AP-MS analysis; Marjorie Cote, Raphae Hafen, Sabina Wirth-Hafen and Désirée Haltiner for their contributions in an undergraduate course; and Peter Gallant, Christian Frei and Hafen lab members for helpful discussions. This work was supported by the Swiss National Science Foundation (SNF) and the ETH Zurich.

\section{Author details}

'Institute of Molecular Systems Biology, ETH Zurich, Wolfgang-Pauli-Strasse 16, 8093 Zurich, Switzerland

${ }^{2}$ Center for Model Organism Proteomes, University of Zurich,

Winterthurerstrasse 190, 8057 Zurich, Switzerland

${ }^{3}$ Urology Research Laboratory, Departments of Urology and Clinical Research, University of Bern, Murtenstrasse 35, 3010 Bern, Switzerland

${ }^{4}$ Institute of Zoology, University of Zurich, Winterthurerstrasse 190, 8057

Zurich, Switzerland

${ }^{5}$ Current address: Department of Medicine, Epigenetics and Progenitor

Cell Keystone Program, Fox Chase Cancer Center, Cottman Avenue 333,

Philadelphia, PA 19111, USA

${ }^{6}$ Current address: Urologische Universitätsklinik, Universitätsspital Basel,

Spitalstrasse 21, 4031 Basel, Switzerland

Submission: 29 August 2009 Revised: 8 December 2009

Accepted: 22 December 2009 Published: 11 February 2010

\section{References}

1. Shibanuma M, Kuroki T, Nose K: Isolation of a gene encoding a putative leucine zipper structure that is induced by transforming growth factor beta 1 and other growth factors. J Bio/ Chem 1992, 267:10219-10224.

2. lida M, Anna CH, Holliday WM, Collins JB, Cunningham ML, Sills RC, Devereux TR: Unique patterns of gene expression changes in liver after treatment of mice for 2 weeks with different known carcinogens and non-carcinogens. Carcinogenesis 2005, 26:689-699.

3. Nakashiro K, Kawamata H, Hino S, Uchida D, Miwa Y, Hamano H, Omotehara F, Yoshida H, Sato M: Down-regulation of TSC-22 (transforming growth factor beta-stimulated clone 22) markedly enhances the growth of a human salivary gland cancer cell line in vitro and in vivo. Cancer Res 1998, 58:549-555

4. Rentsch CA, Cecchini MG, Schwaninger R, Germann M, Markwalder R, Heller $M$, van der Pluijm G, Thalmann GN, Wetterwald A: Differential expression of TGFbeta-stimulated clone 22 in normal prostate and prostate cancer. Int $\mathrm{J}$ Cancer 2006, 118:899-906.

5. Shostak KO, Dmitrenko WV, Vudmaska MI, Naidenov VG, Beletskii AV, Malisheva TA, Semenova VM, Zozulya YP, Demotes-Mainard J, Kavsan VM: Patterns of expression of TSC-22 protein in astrocytic gliomas. Exp Oncol 2005, 27:314-318

6. Ohta S, Yanagihara K, Nagata K: Mechanism of apoptotic cell death of human gastric carcinoma cells mediated by transforming growth factor beta. Biochem J 1997, 324:777-782.

7. Uchida D, Kawamata H, Omotehara F, Miwa Y, Hino S, Begum NM, Yoshida H, Sato M: Over-expression of TSC-22 (TGF-beta stimulated clone-22) markedly enhances 5-fluorouracil-induced apoptosis in a human salivary gland cancer cell line. Lab Invest 2000, 80:955-963.

8. Yu J, Ershler M, Yu L, Wei M, Hackanson B, Yokohama A, Mitsui T, Liu C, Mao H, Liu S, Liu Z, Trotta R, Liu CG, Liu X, Huang K, Visser J, Marcucci G, Plass C, Belyavsky AV, Caligiuri MA: TSC-22 contributes to hematopoietic precursor cell proliferation and repopulation and is epigenetically silenced in large granular lymphocyte leukemia. Blood 2009, 113:5558-5567.

9. Ohta S, Shimekake Y, Nagata K: Molecular cloning and characterization of a transcription factor for the C-type natriuretic peptide gene promoter. Eur J Biochem 1996, 242:460-466.

10. Choi SJ, Moon JH, Ahn YW, Ahn JH, Kim DU, Han TH: Tsc-22 enhances TGF-beta signaling by associating with $\mathrm{Smad} 4$ and induces erythroid cell differentiation. Mol Cell Biochem 2005, 271:23-28.

11. Lee JH, Rho SB, Park SY, Chun T: Interaction between fortilin and transforming growth factor-beta stimulated clone-22 (TSC-22) prevents apoptosis via the destabilization of TSC-22. FEBS Lett 2008, 582:1210-1218.
12. Gluderer S, Oldham S, Rintelen F, Sulzer A, Schutt C, Wu X, Raftery LA, Hafen E, Stocker H: Bunched, the Drosophila homolog of the mammalian tumor suppressor TSC-22, promotes cellular growth. BMC Dev Bio/ 2008, 8:10.

13. Kester HA, Blanchetot $C$, den Hertog J, van der Saag PT, van der Burg B: Transforming growth factor-beta-stimulated clone-22 is a member of a family of leucine zipper proteins that can homo- and heterodimerize and has transcriptional repressor activity. J Biol Chem 1999, 274:27439-27447.

14. Fiol DF, Mak SK, Kultz D: Specific TSC22 domain transcripts are hypertonically induced and alternatively spliced to protect mouse kidney cells during osmotic stress. FEBS J 2007, 274:109-124.

15. D'Adamio F, Zollo O, Moraca R, Ayroldi E, Bruscoli S, Bartoli A, Cannarile L, Migliorati G, Riccardi C: A new dexamethasone-induced gene of the leucine zipper family protects $T$ lymphocytes from TCR/CD3-activated cell death. Immunity 1997, 7:803-812.

16. Delfino DV, Agostini M, Spinicelli S, Vacca C, Riccardi C: Inhibited cell death, NF-kappaB activity and increased IL-10 in TCR-triggered thymocytes of transgenic mice overexpressing the glucocorticoid-induced protein GILZ. Int Immunopharmacol 2006, 6:1126-1134.

17. Delfino DV, Agostini M, Spinicelli S, Vito P, Riccardi C: Decrease of BCl-xL and augmentation of thymocyte apoptosis in GILZ overexpressing transgenic mice. Blood 2004, 104:4134-4141.

18. Riccardi C, Cifone MG, Migliorati G: Glucocorticoid hormone-induced modulation of gene expression and regulation of T-cell death: role of GITR and GILZ, two dexamethasone-induced genes. Cell Death Differ 1999, 6:1182-1189.

19. Asselin-Labat ML, David M, Biola-Vidamment A, Lecoeuche D, Zennaro MC, Bertoglio J, Pallardy M: GILZ, a new target for the transcription factor FoxO3, protects T lymphocytes from interleukin-2 withdrawal-induced apoptosis. Blood 2004, 104:215-223.

20. Ayroldi E, Migliorati G, Bruscoli S, Marchetti C, Zollo O, Cannarile L, D'Adamio F, Riccardi C: Modulation of T-cell activation by the glucocorticoid-induced leucine zipper factor via inhibition of nuclear factor kappaB. Blood 2001, 98:743-753.

21. Ayroldi E, Zollo O, Macchiarulo A, Di Marco B, Marchetti C, Riccardi C: Glucocorticoid-induced leucine zipper inhibits the Raf-extracellular signal-regulated kinase pathway by binding to Raf-1. Mo/ Cell Biol 2002, 22:7929-7941.

22. Mittelstadt PR, Ashwell JD: Inhibition of AP-1 by the glucocorticoidinducible protein GILZ. J Biol Chem 2001, 276:29603-29610.

23. Soundararajan R, Wang J, Melters D, Pearce D: Differential activities of glucocorticoid-induced leucine zipper protein isoforms. J Bio/ Chem 2007, 282:36303-36313.

24. Fiorenza MT, Mukhopadhyay M, Westphal $H$ : Expression screening for Lhx3 downstream genes identifies Thg-1pit as a novel mouse gene involved in pituitary development. Gene 2001, 278:125-130.

25. Hariharan IK, Bilder D: Regulation of imaginal disc growth by tumorsuppressor genes in Drosophila. Annu Rev Genet 2006, 40:335-361.

26. Edgar BA: How flies get their size: genetics meets physiology. Nat Rev Genet 2006, 7:907-916.

27. Harvey K, Tapon N: The Salvador-Warts-Hippo pathway - an emerging tumour-suppressor network. Nat Rev Cancer 2007, 7:182-191.

28. FlyBase [http://flybase.org]

29. Dobens LL, Hsu T, Twombly V, Gelbart WM, Raftery LA, Kafatos FC: The Drosophila bunched gene is a homologue of the growth factor stimulated mammalian TSC-22 sequence and is required during oogenesis. Mech Dev 1997, 65:197-208.

30. Kania A, Salzberg A, Bhat M, D'Evelyn D, He Y, Kiss I, Bellen HJ: P-element mutations affecting embryonic peripheral nervous system development in Drosophila melanogaster. Genetics 1995, 139:1663-1678.

31. Treisman JE, Lai ZC, Rubin GM: Shortsighted acts in the decapentaplegic pathway in Drosophila eye development and has homology to a mouse TGF-beta-responsive gene. Development 1995, 121:2835-2845.

32. Kim J, Lee S, Hwang M, Ko S, Min C, Kim-Ha J: Bunched specifically regulates alpha/beta mushroom body neuronal cell proliferation during metamorphosis. Neuroscience 2009, 161:46-52.

33. Wu X, Yamada-Mabuchi M, Morris EJ, Tanwar PS, Dobens L, Gluderer S, Khan S, Cao J, Stocker H, Hafen E, Dyson NJ, Raftery LA: The Drosophila homolog of human tumor suppressor TSC-22 promotes cellular growth, proliferation, and survival. Proc Natl Acad Sci USA 2008, 105:5414-5419.

34. Brand $A H$, Perrimon N: Targeted gene expression as a means of altering cell fates and generating dominant phenotypes. Development 1993, 
118:401-415.

35. Bischof J, Maeda RK, Hediger M, Karch F, Basler K: An optimized transgenesis system for Drosophila using germ-line-specific phic31 integrases. Proc Natl Acad Sci USA 2007, 104:3312-3317.

36. Gingras AC, Gstaiger M, Raught B, Aebersold R: Analysis of protein complexes using mass spectrometry. Nat Rev Mol Cell Biol 2007, 8:645-654.

37. Glatter T, Wepf A, Aebersold R, Gstaiger M: An integrated workflow for charting the human interaction proteome: insights into the PP2A system. Mol Syst Biol 2009, 5:237.

38. Friggi-Grelin F, Rabouille C, Therond P: The cis-Golgi Drosophila GMAP has a role in anterograde transport and Golgi organization in vivo, similar to its mammalian ortholog in tissue culture cells. Eur J Cell Biol 2006, 85:1155-1166.

39. Hooper JD, Baker E, Ogbourne SM, Sutherland GR, Antalis TM: Cloning of the CDNA and localization of the gene encoding human NRBP, a ubiquitously expressed, multidomain putative adapter protein. Genomics 2000, 66:113-118

40. Lim R, Winteringham LN, Williams JH, McCulloch RK, Ingley E, Tiao JY, Lalonde $J$, Tsai S, Tilbrook PA, Sun Y, Wu X, Morris SW, Klinken SP: MADM, a novel adaptor protein that mediates phosphorylation of the 14-3-3 binding site of myeloid leukemia factor 1. J Biol Chem 2002, 277:40997-41008.

41. Hafen E: Cancer, type 2 diabetes, and ageing: news from flies and worms. Swiss Med Wkly 2004, 134:711-719.

42. Rual JF, Venkatesan K, Hao T, Hirozane-Kishikawa T, Dricot A, Li N, Berriz GF, Gibbons FD, Dreze M, Ayivi-Guedehoussou N, Klitgord N, Simon C, Boxem M, Milstein S, Rosenberg J, Goldberg DS, Zhang LV, Wong SL, Franklin G, Li S, Albala JS, Lim J, Fraughton C, Llamosas E, Cevik S, Bex C, Lamesch P, Sikorski RS, Vandenhaute J, Zoghbi HY, et al:: Towards a proteome-scale map of the human protein-protein interaction network. Nature 2005, 437:1173-1178.

43. Stark C, Breitkreutz BJ, Reguly T, Boucher L, Breitkreutz A, Tyers M: BioGRID: a general repository for interaction datasets. Nucleic Acids Res 2006 34:D535-D539.

44. Bodenmiller B, Malmstrom J, Gerrits B, Campbell D, Lam H, Schmidt A, Rinner O, Mueller LN, Shannon PT, Pedrioli PG, Panse C, Lee HK, Schlapbach R, Aebersold R: PhosphoPep-a phosphoproteome resource for systems biology research in Drosophila Kc167 cells. Mol Syst Biol 2007, 3:139.

45. Ewing RM, Chu P, Elisma F, Li H, Taylor P, Climie S, McBroom-Cerajewski L, Robinson MD, O'Connor L, Li M, Taylor R, Dharsee M, Ho Y, Heilbut A, Moore L, Zhang S, Ornatsky O, Bukhman YV, Ethier M, Sheng Y, Vasilescu J, Abu-Farha M, Lambert JP, Duewel HS, Stewart II, Kuehl B, Hogue K, Colwill K, Gladwish K, Muskat B, et al:: Large-scale mapping of human protein-protein interactions by mass spectrometry. Mol Syst Biol 2007, 3:89.

46. Brunner E, Ahrens $\mathrm{CH}$, Mohanty S, Baetschmann H, Loevenich S, Potthast F, Deutsch EW, Panse C, de Lichtenberg U, Rinner O, Lee H, Pedrioli PG, Malmstrom J, Koehler K, Schrimpf S, Krijgsveld J, Kregenow F, Heck AJ, Hafen E, Schlapbach R, Aebersold R: A high-quality catalog of the Drosophila melanogaster proteome. Nat Biotechnol 2007, 25:576-583.

47. De Langhe S, Haataja L, Senadheera D, Groffen J, Heisterkamp N: Interaction of the small GTPase Rac3 with NRBP, a protein with a kinase-homology domain. Int J Mol Med 2002, 9:451-459.

48. Bard F, Casano L, Mallabiabarrena A, Wallace E, Saito K, Kitayama H, Guizzunti G, Hu Y, Wendler F, Dasgupta R, Perrimon N, Malhotra V: Functional genomics reveals genes involved in protein secretion and Golgi organization. Nature 2006, 439:604-607.
49. Elkins MB, Henry Jj: Isolation and characterization of a novel gene, XMADML, involved in Xenopus laevis eye development. Dev Dyn 2006, 235:1845-1857.

50. Wang H, Sun X, Luo Y, Lin Z, Wu J: Adapter protein NRBP associates with Jab1 and negatively regulates AP-1 activity. FEBS Lett 2006, 580:6015-6021.

51. Mahrour N, Redwine WB, Florens L, Swanson SK, Martin-Brown S, Bradford WD, Staehling-Hampton K, Washburn MP, Conaway RC, Conaway JW: Characterization of Cullin-box sequences that direct recruitment of Cul2Rbx 1 and Cul5-Rbx2 modules to Elongin BC-based ubiquitin ligases. J Biol Chem 2008, 283:8005-8013.

52. Chua JJ, Ng MM, Chow VT: The non-structural 3 (NS3) protein of dengue virus type 2 interacts with human nuclear receptor binding protein and is associated with alterations in membrane structure. Virus Res 2004, 102:151-163

53. Reiling $J H$, Doepfner $K T$, Hafen $E$, Stocker $H$ : Diet-dependent effects of the Drosophila Mnk1/Mnk2 homolog Lk6 on growth via elF4E. Curr Bio/ 2005, 15:24-30.

54. Newsome TP, Asling B, Dickson BJ: Analysis of Drosophila photoreceptor axon guidance in eye-specific mosaics. Development 2000, 127:851-860.

55. Dietzl G, Chen D, Schnorrer F, Su KC, Barinova Y, Fellner M, Gasser B, Kinsey K, Oppel S, Scheiblauer S, Couto A, Marra V, Keleman K, Dickson BJ: A genomewide transgenic RNAi library for conditional gene inactivation in Drosophila. Nature 2007, 448:151-156.

56. Halder G, Callaerts P, Flister S, Walldorf U, Kloter U, Gehring WJ: Eyeless initiates the expression of both sine oculis and eyes absent during Drosophila compound eye development. Development 1998, 125:2181-2191.

57. Hay BA, WolffT, Rubin GM: Expression of baculovirus P35 prevents cell death in Drosophila. Development 1994, 120:2121-2129.

58. Guillen I, Mullor JL, Capdevila J, Sanchez-Herrero E, Morata G, Guerrero I: The function of engrailed and the specification of Drosophila wing pattern. Development 1995, 121:3447-3456.

59. Perkins DN, Pappin DJ, Creasy DM, Cottrell JS: Probability-based protein identification by searching sequence databases using mass spectrometry data. Electrophoresis 1999, 20:3551-3567.

60. Matrix Science [http://www.matrixscience.com]

61. Worby CA, Simonson-Leff N, Dixon JE: RNA interference of gene expression (RNAi) in cultured Drosophila cells. Sci STKE 2001, 2001:PL1.

62. Basler K, Christen B, Hafen E: Ligand-independent activation of the sevenless receptor tyrosine kinase changes the fate of cells in the developing Drosophila eye. Cell 1991, 64:1069-1081.

63. la Cour T, Kiemer L, Molgaard A, Gupta R, Skriver K, Brunak S: Analysis and prediction of leucine-rich nuclear export signals. Protein Eng Des Sel 2004, 17:527-536.

64. ELM [http://elm.eu.org]

65. Nair R, Rost B: Better prediction of sub-cellular localization by combining evolutionary and structural information. Proteins 2003, 53:917-930.

doi:10.1186/jbiol216

Cite this article as: Gluderer S, et al:: Madm (Mlf1 adapter molecule) cooperates with Bunched A to promote growth in Drosophila. Journal of Biology 2010, 9:9. 